\title{
LV-OP-4-3
}

\section{"Anatomic" liver resection performed by approaching the umblical plate for perihilar cholangiocarcinoma (with video)}

\author{
$\underline{\mathrm{Ji} \text { Su KIM }}{ }^{1,2}$, Dai Hoon HAN ${ }^{1,2}$, Kyung Sik KIM ${ }^{1,2}$, Jin Sub $\mathrm{CHOI}^{1,2}$, Gi Hong CHOI ${ }^{* 1,2}$ \\ 'Division of Hepatobiliary and Pancreatic Surgery, Department of Surgery, Yonsei University College of Medicine, Seoul, Korea \\ ${ }^{2}$ Liver Cancer Center, Yonsei Cancer Center, Severance Hospital, Seoul, Korea
}

Introduction: The Anatomic right hepatic trisectionectomy with caudate lobectomy for hilar cholangiocarinoma was reported in 2006. This technique need the umblical plate access. With extension of this technique, anatomic central bisectionectomy can be also performed for the Bismuth type 4 of perihilar cholaniocarcinoma (CCA).

Methods: From 2010 to 2020, anatomic right trisectionectomy $(N=9)$ and anatomic central bisectionectomy $(N=7)$ was performed for the patients with the Bismuth type 4 of perihilar CCA. All anatomic liver resection was performed by approaching the umblical plate, in which the bile ducts of the left lateral section were divided at the left side of the umblical fissure following complete dissection of the umblical plate.

Results: Liver resection was successfully performed, and there was no postoperative mortality. All patients were histologically diagnosed as having CCA. Among the patients who underwent anatomic right trisectionectomy, 4 patients received portal vein embolization and 5 patients did not. R0 resection rate was $60 \% .3$ patients performed portal vein wedge resection. 7 patients died of cancer progression and 2 patients died of biliary sepsis. One patient survived without recurrence for 8 years. 5 patients are under follow-up without recurrence. The remaining one patient recurred after 28 months and is undergoing radiation therapy. There were only four patients had biliary leakage.

Conclusions: Anatomic liver resection that is performed by approaching the umblical plate can be safely and effectively performed even in perihilar CCA. 